\title{
Fatores Preditivos no Tratamento Cirúrgico Vídeotoracoscópico da Hiperidrose Primária
}

\author{
Aluno Aurilio Garcia Lima e Paula (Ciências da Saúde - UniFOA) \\ aur_med@hotmail.com \\ Aluna Lívia Oliveira Delgado Mota (Ciências da Saúde - UniFOA) \\ livinhadelgado@yahoo.com.br \\ Aluno Marco Antônio Ferraz de Barros Baptista (Ciências da Saúde - UniFOA) \\ medicinamarco@yahoo.com.br \\ Aluno Jobson Bortot Filho (Ciências da Saúde - UniFOA) \\ jobinhobf@hotmail.com \\ Aluno Tassius Borsatto Saccomani (Ciências da Saúde - UniFOA) \\ tassiusbs@terra.com.br \\ Prof. Doutor Mauro Tavares (Ciências da Saúde - UniFOA) \\ mauro.tavares@foa.org.br
}

\section{Resumo}

Este artigo apresenta e discute os fatores preditivos no tratamento cirúrgico videotoracoscópico da hiperhidrose primária através do estudo de vinte pacientes e a análise dos resultados obtidos com os mesmos.

Palavras Chave: Hiperhidrose, Tratamento Cirúrgico Videotoracoscópico.

\begin{abstract}
This article presents and argues the preditive factors in the thoracoscopic surgical treatment of hyperhidrosis primary through the study of twenty patients and the analysis of the results.
\end{abstract}

Key-Words: hyperhidrosis, thoracoscopic surgical.

\section{INTRODUÇÃO}

O suor é responsável pelo controle da temperatura corpórea, que é mediado pelo Sistema Nervoso Simpático. A hiperatividade desse sistema 
leva ao aumento das atividades das glândulas sudoríparas gerando uma sudorese excessiva, condição conhecida como hiperidrose. Sua incidência acomete 0,5 a $1 \%$ da população, sendo considerada uma condição clínica freqüente (COELHO, 2006). O Sistema nervoso humano é composto por uma região central e uma periférica. Anatomicamente ele divide-se em Sistema Nervoso Visceral, que tem como objetivo a manutenção da homeostase visceral, e em Sistema Nervoso Somático que objetiva controlar a relação do indivíduo com o meio. Ambos são constituídos por fibras aferentes (conduzem informação periférica para o centro) e eferentes (condução do impulso do centro para a periferia). O componente eferente do sistema nervoso visceral é denominado de Sistema Nervoso Autônomo (SNA) que se divide em Sistema Nervoso Simpático e Sistema Nervoso Parassimpático (CARPENTER, 1991). As fibras pré-ganglionares do Sistema nervoso Parassimpático têm origem em núcleos motores de nervos cranianos presentes no tronco encefálico ou segmento sacromedular. Já os corpos celulares das fibras pré-ganglionares do Sistema Nervoso Simpático originam-se no segmento toracolombar T1 a L2 ou L3 e atingem a porção periférica através das cadeias paravertebrais (CARPENTER, 1991). Em determinadas situações como calor intenso, ansiedade e fortes emoções- os centros termorreguladores do hipotálamo são ativados e, via SNA, estimulam a secreção das glândulas sudoríparas écrinas, resultando na perda de calor através do suor. A hiperexcitação das fibras préganglionares do SNA causam a Hiperhidrose (GUYTON, 1981). A hiperhidrose pode ser primária ou secundária. A primária não tem causa conhecida (genética), já a secundária pode ocorrer por condições como hipertireoidismo, distúrbios psíquicos, obesidade, etc. Pode ser axilar, palmar, plantar, facial (região frontal principalmente e couro cabeludo), dorso e região inguinal. A hiperhidrose é uma condição que causa limitação no convívio social e profissional do indivíduo, podendo inclusive gerar um odor fétido quando em muito excesso, além de favorecer a proliferação de fungos e bactérias (LEÃO, 1999). Existe uma forma de tratamento clínico com toxina botulínica que pode ser aplicado no local e tem ação temporária, encarecendo o tratamento (COELHO, 2006). Como tratamento cirúrgico, a Simpatectomia que é a secção da cadeia ganglionar, foi proposta e tornou-se $1^{a}$ opção $p /$ tratamento da hiperhidrose, além de outras indicações como Doença de Raynaud, Síndrome do QT largo (COELHO, 2006). Está contra-indicada na hiperhidrose secundária , seqüelas de doenças pleurais como tuberculose ou empiema pleural, IMC $>25$ pois o resultado é ruim. O efeito colateral mais comum é a sudorese reflexa que é um suor excessivo em regiões não denervadas, o que levou os cirurgiões a procurarem cirurgias mais seletivas. O primeiro gânglio parassimpático é o T1 que é responsável pelo suor da face, mãos e, em 
menor extensão, axila. T2 controla secreção das mãos, face, couro cabeludo, ombros e parte anterior do tórax, acém do rubor facial. T3 controla sudorese de mãos, axila, ombros e parte anterior e posterior do tórax. T4 controla mãos e axila. Nas primeiras simpatectomias a exérese de T2 era realizada para hiperhidrose crânio-facial e rubor facial, T3 para hiperhidrose palmar, T3 e T4 para hiperhidrose axilar associada ou não à palmar (CELHO, 2006). Segundo LYRA (2006) as cirurgias são, na maioria das vezes, curativas. 90\% melhoram da hiperhidrose palmar, axilar, facial, em couro cabeludo ou rubor facial. Entretanto, 85\% dos pacientes relataram hiperhidrose compensatória após período superior a 1 ano, sendo mais severa nos pacientes submetidos à simpatectomia em T2. RIET e cols. (2001) realizaram 14 simpaticotomias com cauterização cranial e caudal a T3. A cirurgia mostrou-se eficaz. FURIAN (2005), constatou que $38,1 \%$ dos 244 pacientes submetidos a simpaticotomia cranial e caudal a T4 apresentam algum grau de hiperhidrose compensatória, porém nenhum deles a considerou insuportável.

Assim, desenvolvemos este estudo analisando a localização da hiperidrose primária, o sexo, o índice de massa corpórea, a remissão dos sintomas no pós-operatório com menos de seis meses e com mais de seis meses, a incidência de hiperidrose reflexa, a dor pós-operatória e o índice de satisfação dos pacientes com os resultados proporcionados pela operação.

\section{MATERIAL E MÉTODO}

Foram estudados, retrospectivamente, 20 pacientes oriundos do Serviço de Cirurgia Torácica do Hospital Municipal do Retiro (HMR), submetidos à simpaticotomia videotoracoscópica, por hiperidrose crânio-facial, axilar, axilo-palmar e palmar. Esses pacientes foram submetidos à entrevista mediante o preenchimento de questionário (Anexo). A técnica operatória empregada em todos os pacientes foi: utilizou-se 02 mini-incisões em cada hemitórax : uma na linha axilar anterior (junto ao ôco axilar) e outra na linha hemiclavicular, sendo na mulher no sulco submamário, e no homem periareolar. Pela incisão periareolar, ou submamária, posicionamos um trocart de $10 \mathrm{~mm}$, e pela incisão axilar, um trocart de $5 \mathrm{~mm}$. A cadeia simpática foi abordada e seccionada bilateralmente, em nível de acordo com a indicação: hiperidrose crânio-facial, nível T2; hiperidrose axilar, nível T4; hiperidrose palmar, nível T3; hiperidrose axilo-palmar, nível T3 e T4. Imediatamente após a simpaticotomia, e antes de promovermos a expansibilidade pulmonar, instilamos Bupivacaína 0,5\% sem vasoconstrictor nos locais cauterizados. Todos os pacientes ficaram internados, no máximo, 24 h. Em todos eles administramos 50 mg de diclofenaco sódico a cada 8 horas. 


\section{RESULTADOS}

Encontramos, nos 20 casos estudados, a seguinte incidência de hiperidrose: um (5\%) com hiperidrose palmar isolada, dois (10\%) com hiperidrose palmar e axilar, dois (10\%) com hiperidrose axilar e palmar associada com facial ou abdominal, três (15\%) com palmar, plantar e axilar associadas, seis (30\%) com palmar e plantar, seis (30\%) com axilar isolada (gráfico 1).
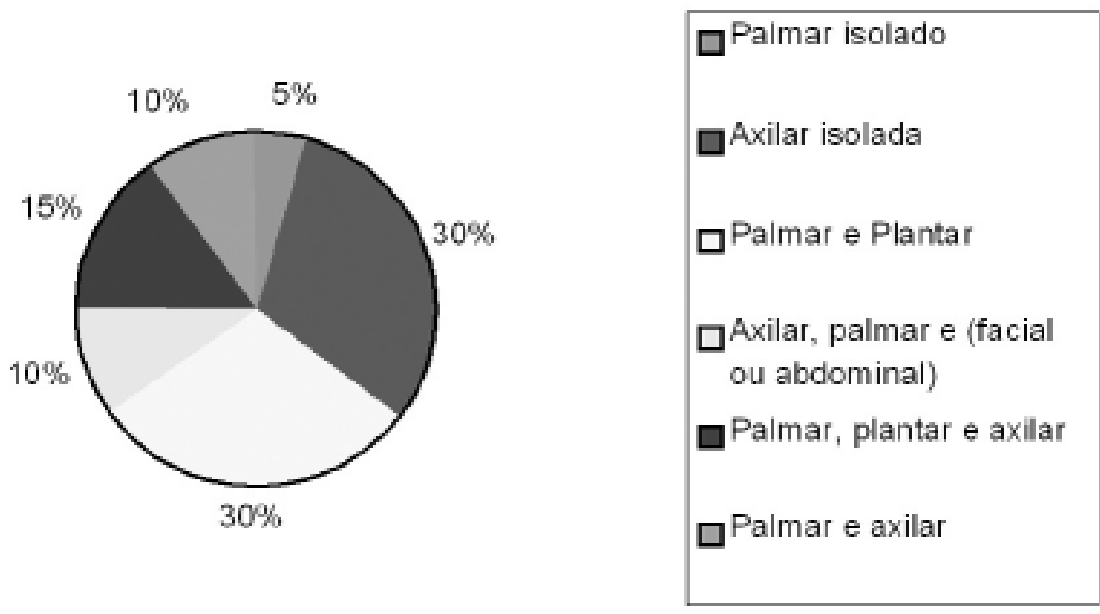

Gráfico 1 - Tipos de hiperidrose encontradas em nosso estudo.
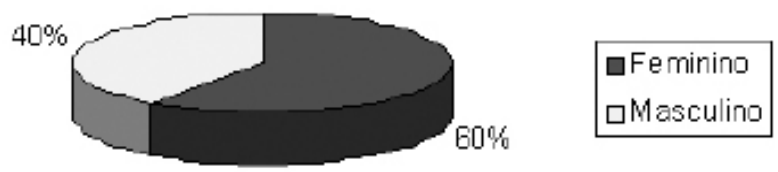

Gráfico 2 - Distribuição dos pacientes de acordo com o sexo.

Em relação ao Índice de Massa Corporal (IMC) do grupo estudado, obtivemos 5 pacientes (25\%) com baixo peso, 10 (50\%) com IMC normal, 3 (15\%) com sobrepeso e 2 (10\%) com obesidade (Gráfico 3).

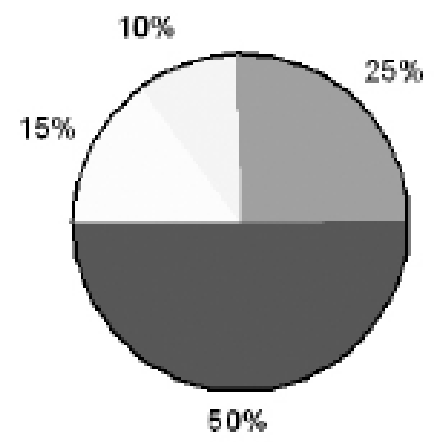

$\square$ Baixo Peso
$\square$ Normal
$\square$ Sobrepeso
$\square$ Obeso

Gráfico 3 - Distribuição dos pacientes de acordo com o IMC. 
Em relação à remissão do quadro de hiperidrose no pós-operatório, 65\% (13 pacientes) relataram melhora parcial ou completa. Sete pacientes (35\%) não obtiveram melhora (gráfico 4).
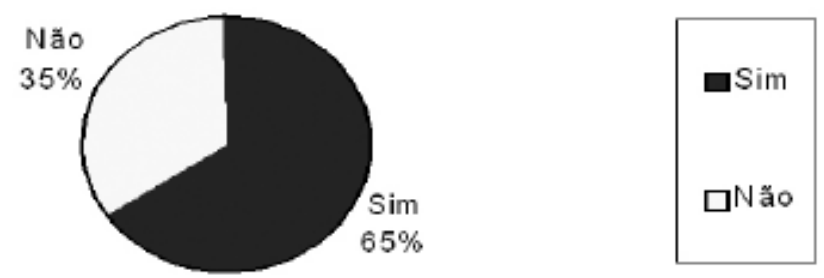

Gráfico 4 - Porcentagem de remissão dos sintomas no pós-operatório.

A incidência de hiperidrose reflexa foi verificada em 17 pacientes (85\%). Somente 3 pacientes (15\%) não tiveram esta complicação (gráfico 5). As regiões mais acometidas pela hiperidrose reflexa foram: abdome, costas e coxas. Em 10 pacientes (50\%) as regiões acometidas foram a anterior do abdome, tórax anterior e posterior e axila; só o abdome em 2 pacientes (10\%), a coxa isoladamente em 2 pacientes (10\%), a região plantar associado às costas ou face , em 2 pacientes (10\%), o tórax e o abdome em 4 pacientes (20\%). (Gráfico $6)$.

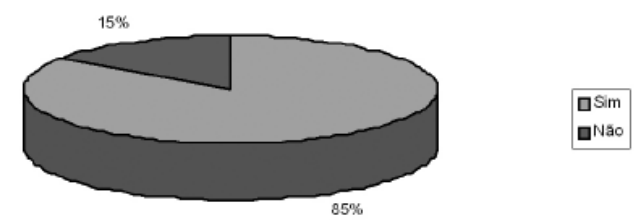

Gráfico 5 - Incidência da hiperidrose reflexa.
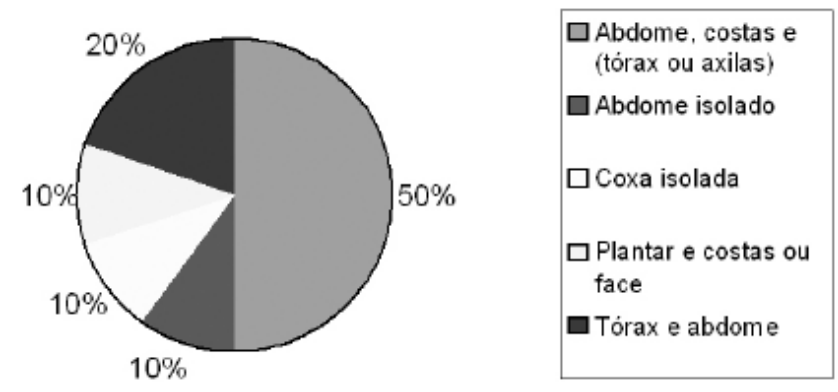

Gráfico 6 - Regiões acometidas pela hiperidrose reflexa.

Analisando o incômodo na cicatriz cirúrgica, somente 3 pacientes (15\%) relataram que as cicatrizes cirúrgicas incomodaram. Por outro lado, 17 pacientes (85\%) não se incomodaram (gráfico 7). 


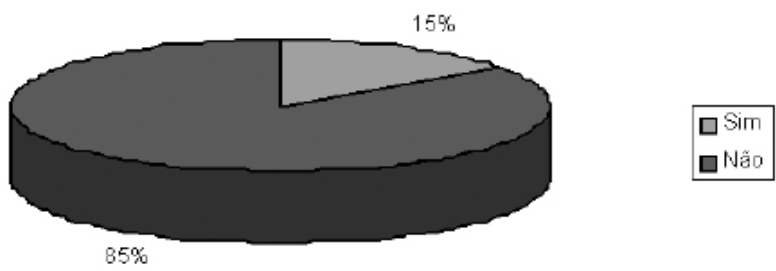

Gráfico 7 - Incidência de incômodo na cicatriz cirúrgica.

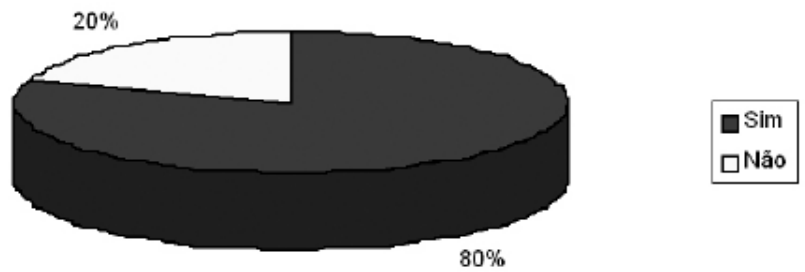

Gráfico 8 - Satisfação com os resultados pós-operatórios.

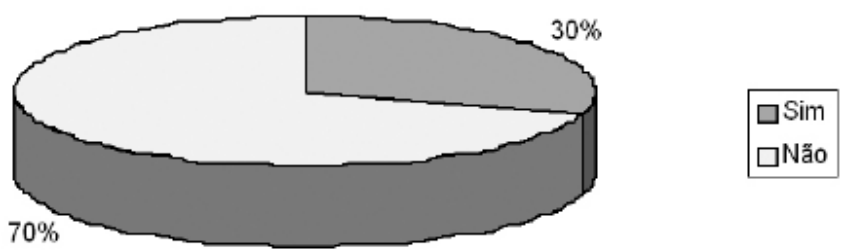

Gráfico 9 - Incidência da dor pós-operatória.

Os pacientes também foram estudados de acordo com o tempo de pós-operatório e a melhora dos sintomas. Os pacientes com acompanhamento menor que 6 meses somaram cinco (5). Desses, 3 pacientes (60\%) relataram melhora dos sintomas e 2 (40\%) não melhoraram (Gráfico 10). Os pacientes com acompanhamento maior que 6 meses somaram 15. Assim, tivemos 10 pacientes (67\%) referindo melhora e 5 pacientes (33\%) sem melhora (Gráfico 11).

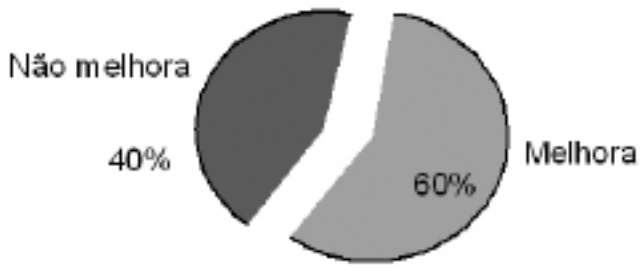

$\square$ Melhora

Năo melhora

Gráfico 10 - Resultados com menos de 6 meses de pós-operatório. 
Năo Melhora

$33 \%$

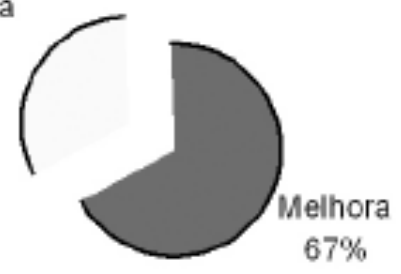

$\square^{\text {Melhora }}$
$\square^{\text {Năo Melhora }}$

Gráfico 11 - Resultados com mais de 6 meses de pós-operatório.

\section{DISCUSSÃO}

Assim como na maioria da literatura (STORI, 2006; WILSON, 2005) o sexo feminino predominou (60\%). É possível que um dos motivos da predominância do sexo feminino seja o constrangimento que a doença acarreta na vida social das pacientes e que as mulheres tenderiam a incomodar-se mais com essa situação, acarretando num aumento na procura pela terapêutica cirúrgica.(STORI, 2006). A idade dos pacientes variou de 13 a 51 anos (média de 32 anos). Na literatura, a idade média no momento da cirurgia variou de 14,1 a 31 anos (GOSSOT, 2001; WILSON, 2005; STORI, 2006). Observamos ,em nossa pesquisa, que o início médio dos sintomas foi aos 12,2 anos. A Hiperidrose axilar isolada foi encontrada em 6(seis) pacientes, enquanto a maior parte dos pacientes apresentava hiperidrose combinada. A Hiperidrose palmar isolada foi encontrada em apenas um paciente (5\%). Encontra-se na literatura a possibilidade de ocorrência de complicações, ainda que incomuns, como a síndrome de Claude Bernard-Horner, quilotórax e hemotórax , presentes em torno de 1,8\% (LEÃO, 2003). Nenhuma dessas complicações foram observadas no grupo estudado. A incidência de dor torácica de forte intensidade esteve presente em 6 (30\%) pacientes - dados semelhantes aos obtidos da literatura (DUMONT, 1997; BYRNE, 1990). Podem apresentar dor por neurite intercostal, por manipulação da pleura parietal e por eletrocoagulação do periósteo costal durante a secção do tronco simpático em seu trajeto sobre a costela. A neuralgia intercostal ocorre possivelmente por trauma ocasionado pela manipulação ao longo do espaço intercostal. Em termos de resultado da cirurgia, dos pacientes que foram submetidos ao procedimento e entrevistados num período menor do que 6 meses após a cirurgia, dois (10\%) queixaram-se não ter havido melhora dos sintomas. Já o grupo de pacientes entrevistados com mais de 6 meses de pós-operatório cinco (25\%) relataram ausência de melhora. Dos seis (30\%) pacientes que apresentavam hiperidrose axilar isolada ou combinada, quatro (20\%) relataram não melhora do sintoma axilar, o que aumentou a estatística dos pacientes insatisfeitos com a cirurgia. Além desses, um (5\%) paciente que apresentava hiperidrose combinada também relatou que os sintomas axilares não cessaram. Publicações recentes demonstram que a simpaticotomia para hiperidrose axilar tem resultado inferior do que quando realizada para hiperidrose plantar, por haver diferentes tipos histológicos de glândulas na axila (De CAMPOS, 2003; TALARICO, 2003). Dos 20 pacientes estudados observou-se sobrepeso ou obesidade em 5 (25\%) e destes, três não obtiveram melhora pós-operatória nem 
satisfação com o procedimento realizado. Foi avaliado o grau de satisfação dos pacientes e ,de todo o grupo estudado, quatro (20\%) tiveram insatisfação total com o procedimento, sendo que todos eram portadores de hiperidrose axilar isolada. Já os que obtiveram satisfação com os resultados somaram 80\% (16 pacientes) o que reforça os dados da literatura que diz que entre 72 e $94 \%$ dos pacientes ficam satisfeitos com a cirurgia (CAMARGO, 2003; WILSON, 2005). Analisando a sudorese compensatória (SC) ocorrida em 85\% dos casos, o local de maior acometimento foi o abdome (50\%), seguido da região dorsal e de outras áreas, como tórax, coxa, plantar e face. Na literatura, a incidência de SC varia de 32 a 86\%, e sendo os locais mais acometidos as costas (63\%), coxas (32\%) e tórax (31\%) (BYRNE, 1990; LIN, 1999).

\section{CONCLUSÃO}

A hiperidrose primária é uma anormalidade que, sem dúvida alguma, leva a um impacto social muito grande, principalmente quando se trata de meninas, jovens, em processo de inclusão no mercado de trabalho ou na fase da adolescência. O relacionamento com outras pessoas é o principal fator a causar constrangimentos aos pacientes portadores de hiperidrose primária. Os tratamentos clínicos, além de caros, não são efetivos. O tratamento cirúrgico é a modalidade definitiva de tratamento para essa doença. Contudo, a hiperidrose reflexa é uma realidade que deve ser encarada com muita seriedade e parcimônia na indicação do tratamento cirúrgico. Fatores como o índice de massa corpórea transformou-se de contra-indicação relativa para contra-indicação obsoluta, face à alta incidência, neste grupo de pacientes, de hiperidrose reflexa; a idade é outro fator que passou a se comportar como o índice de massa corpórea.

Por fim, é preciso que critérios de inclusão no tratamento cirúrgico sejam bem delimitados, assim como os fatores preditivos positivos e negativos também sejam bem estabelecidos para que o tratamento cirúrgico da hiperidrose primária seja o mais desprovido possível de recidivas, complicações e insatisfação por parte dos pacientes.

\section{Referências Bibliográficas}

BYRNE, J., WALSH, T.N., HEDERMAN, W.P. Endoscopic transthoracic electrocautery of the sympathetic chain for palmar and axillary hyperhidrosis. $\mathrm{Br} \mathrm{J}$ Surg. 1990;77:1046-9.

CAMARGO, J.J., TEDDE, M.L. Simpatectomia. In: Almeida ART, Hexsel DM. Hiperidrose e toxina botulínica. São Paulo: Know-How; 2003. p. 125-35.

CARPENTER, M.B. Core Text of Neuroanatomy. Baltimore, Ed. Williams \& Wilkins, 1991. 
COELHO,M.Hiperhidrose.Paraná,jun2006.http://www.marloscoelho.com.br/conteudo_hi perhidrose.php?acao=hiperhidrose\&area=hiperhidrose_completa\&idioma=1] Acesso em 22 de Setembro de 2006.

De CAMPOS, J.R.M., KAUFFMANN, P., WEREBE, E.D.C., ANDRADE FILHO, L.O., et al. Quality of life, before and after thoracic sympathectomy: report on 378 operated patients. Ann Thorac Surg. 2003;76:886-91.

DOOLABH, N., HORSWELL, S., WILlIAMS, M., HUBER, L., PRINCE, S., MEYER, D.M et al. Thoracoscopic sympathectomy for hyperhidrosis: indications and results. Ann Thorac Surg. 2004;77:410-4.

DUMONT, P., HAMM, A., SKROBALA, D., ROBIN, P., TOUMIEUX, B. Bilateral thoracoscopy for sympathectomy in the treatment of hyperhidrosis. Eur $\mathrm{J}$ Cardiothorac Surg. 1997;11:774-5.

FURIAN, M.B. Simpatectomia Videotoracoscópica e Hiperhidrose Reflexa Pós Operatória. Boletim Científico da Sociedade Brasileira de Cirurgia Torácica, ano II n.4, 2005.

GOSSOT, D., GALLETA, D., PASCAL, A. et al. Lond-Term results of endoscopic thoracic sympathectomy for upper limb hyperhidrosis. Ann Thorac Surg 75: 1075-9, 2003.

GUYTON, A.C. Fisiologia Humana. 5ª ed., Rio de Janeiro, Ed. Interamericana, 1981.

LEÃO, L.E.V., De OLIVEIRA, R., SZULC, R., MARI, J.J., CROTTI, P.L., GONÇALVES, J.J. Role of video-assisted thoracoscopic sympathectomy in the treatment of primary hyperhidrosis. Sao Paulo Med J. 2003;121:191-7.

LEÃO, L.E.V.; GIUDICI,R. Simpatectomia Torácica por Videotoracoscopia: tratamento atual da hiperhidrose palmar. Revista Jovem Médico, maio 1999.

LIN, C.C., WU, H.H. Endoscopic T4-Sympathectomy block by clamping(ESB4) in treatment of hyperhidrosis palmaris et axillaris - experiences of 165 cases. Annales Chirurgiae et Gynaecologiae 90:167-169, 2001.

LIN, C.C., TELERANTA, T. Lin-Telaranta classification: The importance of different procedures for different indications in sympathetic surgery. Annales Chirurgiae et Gynaecologiae 90: 161-166, 2001.

LYRA, R.B. e SALVESTRO, J.E.S. Informação aos pacientes com transpiração excessiva. São $\quad$ Paulo, 2006. $\quad$ Disponível em [http://www.geocities.com/manuseio/hiperidrose.html]. Acesso em 22 de Setembro de 2006. 
REISFELD, R., NGUYEN, R., PNINI, A. Endoscopic thoracic sympathectomy for hyperhidrosis: experience with both cauterization and clamping methods. Surg Laparosc Endosc Percutan Tech. 2002;12:255-67.

RIET, M., SMET, A., KUIKEN, H, KAZEMIER, G., BONJER, H.J. Prevention of compensatory hyperhidrosis after thoracoscopic sympathectomy for hyperhidrosis. Surg Endosc 15:1159-1162, 2001.

STORI, Jr., SOUZA, W. Bloqueio por clipagem de gânglios simpáticos torácicos no tratamento da hiper-hidrose. An. Bras. Dermatol., Out 2006, vol.81, no.5, p.425-432

TALARICO FILHO, S., NASCIMENTO, M.M. Avaliação do paciente com hiperhidrose. In: Almeida ART, Hexsel DM. Hiperidrose e toxina botulínica. São Paulo: KnowHow; 2003. p.57-9.

WILSON, M.J., MAGEE, T.R., GALLAND, R.B., DEHN, T.C. Results of thoracoscopic sympathectomy for the treatment of axillary and palmar hyperhidrosis with respect to compensatory hyperhidrosis and dry hands. Surg Endosc. 2004. [acesso $03 \mathrm{Fev} 2005$ ] Estudo da microbiota fúngica na mucosa conjuntival de acadêmicos da área das Ciências da Saúde do Centro Universitário de Volta Redonda - UniFOA

Informações bibliográficas:

Conforme a NBR 6023:2002 da Associação Brasileira de Normas Técnicas (ABNT), este texto científico publicado em periódico eletrônico deve ser citado da seguinte forma:

PAULA, A. G. L.; MOTA, L. O. D; BAPTISTA, M. A. F. B.; BORTOT FILHO, J.; SACCOMANI, T. B.; SOUZA, M. C. T. Fatores Preditivos no Tratamento Cirúrgico Vídeotoracoscópico da Hiperidrose Primária. Cadernos UniFOA , Volta Redonda, ano 2, nº. 3, mar. 2007. Disponível em:

$<$ http://www.unifoa.edu.br/pesquisa/caderno/edicao/03/121.pdf> 